\title{
On how to design smart energy-efficient buildings
}

\author{
Donatella Sciuto \\ Politecnico di Milano \\ donatella.sciuto@polimi.it
}

\author{
Alessandro A. Nacci \\ Politecnico di Milano \\ alessandro.nacci@polimi.it
}

\begin{abstract}
Smart spaces are environments such as apartments, offices, museums, hospitals, schools, malls, university campuses, and outdoor areas that are enabled for the cooperation of objects (e.g., sensors, devices, appliances) and systems that have the capability to self-organize themselves, based on given policies. Since they can be used for an efficient management of the energy consumption of buildings, there is a growing interest for them, both in academia and industry. Unfortunately, nowadays, these systems are still designed manually with ad-hoc solutions. As a consequence, a huge effort has to be spent for each new smart building. Within this context, aim of this work is to propose a methodology to automate the design process of such smart spaces. The paper presents an overview of the design flow implemented to support the design of scalable architectures for energy aware smart spaces.
\end{abstract}

Keywords-Smart buildings, energy efficiency, design methodologies

\section{INTRODUCTION}

Considering the continuous development of the sensor/actuator technologies in the last few years, and the incessant growth of their applications in almost all the aspects of our everyday life, the so called "smart spaces" are something feasible and actually available. Smart spaces are environments such as apartments, offices, museums, hospitals, schools, malls, university campuses, and outdoor areas that are enabled for the cooperation of objects (e.g., sensors, devices, appliances) and systems that have the capability to selforganize themselves, based on given policies [14]. The interesting point is that this technology can be also used in order to enable the automatic management of buildings and spaces power consumption, thus drastically increasing their energy efficiency. Most of the components to realize such smart spaces are already available in the market, but unfortunately it is still necessary to perform a manual design (e.g., defining the number and the location of each component and the communication protocols among them) in order to make them working properly in real-world scenarios. This implies that a huge effort is required every time a new smart space (e.g., a smart building for an exhibition) has to be built. In this context, it would be fascinating to explore the possibility of defining a methodology and a set of design tools to automate the creation of smart spaces, thus making their design much easier, faster and cheaper. In particular, we aim at developing a methodology that, starting from the high-level representation of the target environment and of the desired functionalities of the target smart space, would make it possible to automatically derive the software and the specification of both the hardware and the communication infrastructure that are best suited to implement the smart space itself.

\section{SMART BuILDINGS}

Smart building are the next generation of living and working environments. Their peculiarity is the use of ICT to coordinate the different aspects of the building to increase the user comfort, the energy efficiency and the user safety.

\section{A. User comfort}

The use of ICT in smart buildings can deeply improve the occupants' comfort automatically controlling the environment temperature, humidity, brightness, and fitting all these parameters on the single user needs. Just to make some examples, a smart building must be able to turn on and off automatically the lights when a room is used or empty; it has to dynamically adjust the room temperature with respect to the weather condition and the current user living the space. In a smart buildings, the appliances has to be ready to be used when the user need them, without requiring the user to think about their power status.

\section{B. Energy Efficiency}

Alongside with the user comfort, a smart building has to carefully manage how it uses energy to accomplish all the tasks. For instance, knowing the user needs, it is possible to tune the Heating, Ventilation and Air Conditioning (HVAC) system in order to heat only for the needed time, without wasting energy resources. Moreover, a smart building is generally thought to be part of a smart grid, the next generation electric grid: within this context, the building has to be able to sense and forecast its energy needs and to reply to energy saving commands coming from the smart grid.

\section{User Safety}

Another important aspect is the user safety: thanks to its smartness, a building has to help the occupants during different emergencies like fires or earthquakes: it is possible to imagine advanced alarm systems and modern firefighting equipment 
able to understand the context and to help the aids to be more effective.

\section{SMART BuILDINGS DESIGN COMPLEXITY}

In order to achieve all the goals presented in the previous section, a smart building has to rely on a complex and interconnected hardware infrastructure composed of sensors and actuators. In fact, to realize these smart spaces, dozens of distributed computation, perception and actuation modules are usually adopted [15]. Sensors gather a huge quantity of information that must be elaborated from the computation nodes and then efficiently transmitted to the actuators. Moreover, in order to make these spaces easy to use to common users the so-called eco-feedback technology are in great need ([16][17]): in fact, they aim both at designing efficient and intuitive mechanisms to display energy consumption and at studying the related consumers' awareness. The proposed representation of generic smart space architecture is shown in Figure 1.

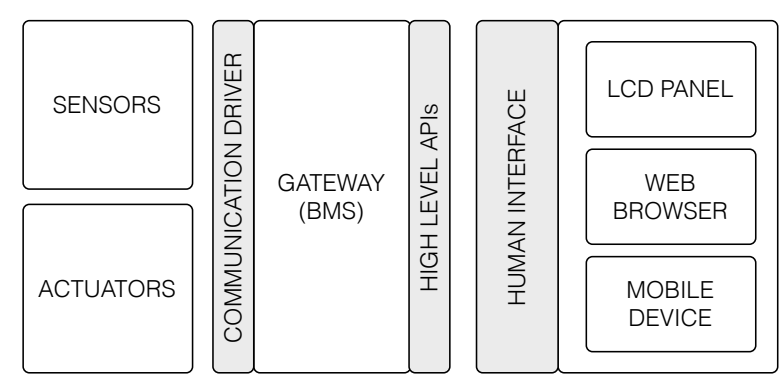

Figure 1. Smart space general architecture

The system is composed of different sensors and actuators coordinated by a central system, the Energy Box, which is also responsible for the interfaces needed for visualization purposes. The Energy Box can be intended as an embedded device responsible for gathering data from the different sensors in the smart space and for the actuation through the available actuators. Since potentially, many of these devices must be installed in a building, they must be low power and, consequently, they do not have a huge computational capability. As a consequence, the transfer function that has as input the data coming from the sensor and as output the actions performed by the actuators can be computed directly in situ by the energy box only for simple rules. Whenever complex computations are required for the computation of the transfer function, it can be retrieved from a remote server. Another possible idea is to split the computation among all the energy boxes available in the building, i.e. by means of distributed computation. As shown in Figure 2, an energy box, working as a Local Building Management System (LBMS), stores locally with a cache mechanism - only the data it needs for the computation of the policies. Then, if necessary, data is sent to a remote server (the stat-server) and stored for statistics and data mining.

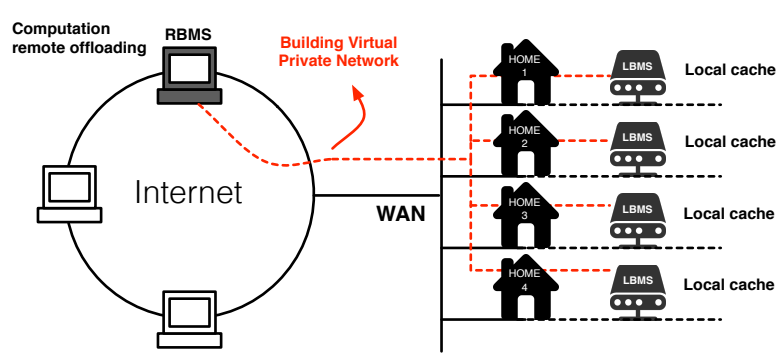

Figure 2. Distributed building management system architecture

The energy box has also a standard communication interface that allows the users to access the gathered information by mobile devices (tablet, smartphones), by installed panels or by a common web browser. The graphical user interface, if existing, is managed by an interface server that reads the data from the energy boxes or from the statserver. These aspects are represented in Figure 3.

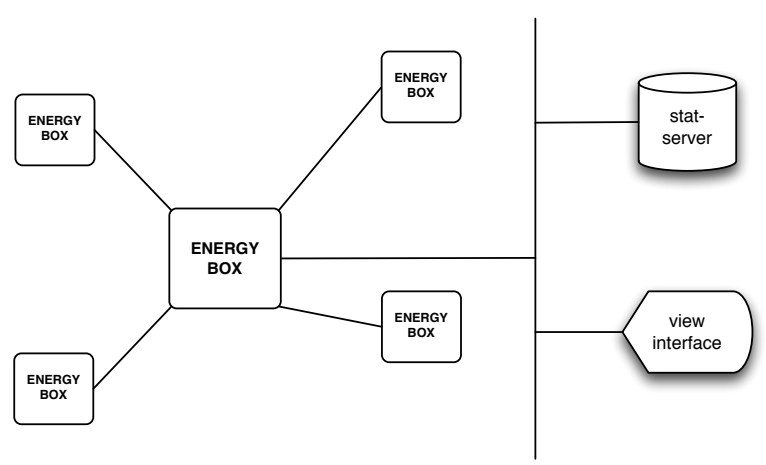

Figure 3. Energy boxes hierarchical architecture

Currently, many different vendors provide such components, and often communication protocols are not standard; moreover, the components themselves present different features even if they are designed to pursue the same goals (two temperature sensors by two different vendors can have a different granularity, a different measure error an so on). This high variability in the technological substrate, lead to a very hard system integration that has currently to be performed in a custom way with respect to the single considered building. In order to manage such a complexity, methods are in great need to face the different situations the building has to face. Moreover, these methods are the baseline for the creation of design tools that can support the design of such smart buildings in a standard way.

\section{A DEVElopment Suite}

In order to simplify the design of such smart buildings, we are working on the definition of an automated design suite that is able to create the actual and working implementation of the architecture described above. The tool receives as input a 


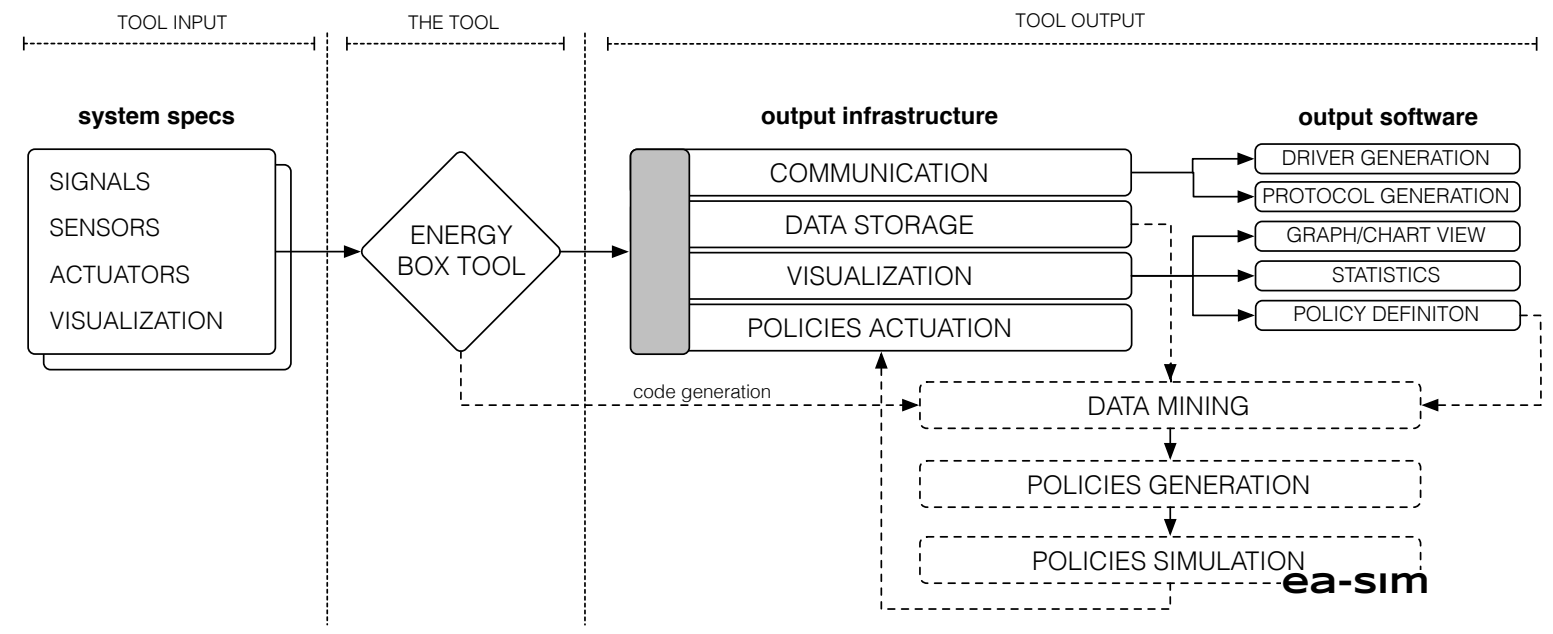

Figure 4. Smart space design flow

description of the sensors and of the actuators and also a description of the signals that need to be considered; from the provided inputs, it automatically generates the software and communication infrastructure for the management of the energy aware smart space.

Figure 4 shows a very high level view of the proposed design flow. The input is the specification of the signals to be monitored or used for the actuation, sensors description, actuators description and the definition of the data to be visualized. Taking as input these data, the design flow automatically generates:

- The communication infrastructure among all the nodes of the system (driver and protocols);

- The data storage server, setting up also the database and the needed interfaces to read and write;

- All the visualization software (for panels, mobile devices, web browsers);

- the mechanisms needed for the actuation of the policies.

Moreover, the tool has to generate the code to perform a custom data mining to automatically generate energy saving policies based on the users behaviors. The data mining can be performed on two different possible sources:

- On the data stored into the database, the statserver, i.e. the data coming from sensors and actuators;

- On the old policies defined by the users.

The main difference between the two approaches is that, the first one does not need a pre-defined set of policies chosen by the users since it reads directly from the raw environmental data, while the second one needs it.

\section{POLICY Managment}

The design and the management of "smart spaces" is not a trivial task, especially because of their intrinsic complexity. For instance, modern commercial buildings often employ a centralized Heating, Ventilation, and Air Conditioning (HVAC) system in addition to lighting, fire safety, elevators and security systems. In spite of this complexity, a correct and efficient energy management is of paramount importance, especially when considering that buildings contribute to the $70 \%$ of the total energy consumption of an electrical grid [1] and up to $45 \%$ of this energy consumption is due to heating, cooling, and lighting [2]. For this reason, it is common for modern buildings to employ a centralized Building Management System, which tries to improve the management of the different subsystems in order to optimize the overall energy consumption of the building. A generic BMS can be thought as the composition and the coordination of different distributed element, the aforementioned Energy Boxes. However, the BMS solutions already available in the market [3][4][5] currently aim only at supporting the building managers for what concerns their daily supervision tasks, while delegating specialized personnel for the maintenance and for specialized tasks related to each subsystem.

On the contrary, it has been recently shown in [6] that this user-oriented customization can also help in improving the energy efficiency of the building itself. For this reason, the idea of providing the users with a tool to express their needs, requirements and preferences has been discussed in several recent research works that deal with the improvement of buildings energy efficiency (e.g.,[7][8][9][10][11][12]). However, energy efficiency is not the only factor in which users can be interested in: for instance, it could be possible to attract their attention (and thus their collaboration) by providing them with better services or by increasing their living and working comfort.

In this context, we aim at dealing with the two complementary issues that still have to be solved in order to enable a widespread adoption of smart spaces in our everyday life: on the one hand, the design of their hardware infrastructure (including tasks such as a deep trade-off exploration and the validation/simulation of the different 
feasible solutions), while, on the other, the rising of the abstraction level of their management policies (including the definition of a user-friendly interface and specification language, and the implementation of an automatic satisfiability checking algorithm), in order to let the users easily and seamlessly interact with the surrounding environment.

\section{BUILDING SimULATION}

In order to test the policies and to forecast the behavior of the environment, a simulation framework is needed into the design suite. Within this context, the EA-SIM [13][13] simulator can be used. EA-SIM is a novel, open-source and ongoing framework for the design and the simulation of energy aware smart spaces that is based on SystemC TLM-2.0 as representation abstraction. This allows both a functional validation of the generated systems, but also an analysis of the non-functional properties, such as performance and power consumption at different levels of abstraction. Moreover, it can be also integrated with the simulation of the physical environment (e.g., thermal trends, power consumption) to validate the decisions taken by the control units with respect to the information gathered by the sensors. In particular, EA-SIM offers an extensible component library that allows modeling of the different elements of a smart space: the control units, the computational nodes, the appliances, along with their sensors and actuators. Moreover, it is also possible to associate local batteries with each appliance and control their usage to reduce peaks in the electrical absorption. To the best of our knowledge, this is the first framework that allows a systemlevel simulation of such kind of environments.

\section{CONCLUDING REMARKS}

In this paper we discussed the need for a methodology for the design of energy aware smart spaces. Design methodologies are in fact in great need to cope with the complexity of such systems, that derives from the huge number of sensors, actuators and control elements that have to work in a synergic way in order to realize future smart spaces able to increase the user wellbeing, safety and energy efficiency. We proposed a design flow that we are currently developing, able to get as input a high level description of a building and to generate the implementation of the smart building ICT infrastructure. This design flow includes policy management mechanisms and a simulation framework to make possible the exploration phase required to evaluate different technical solutions.

\section{REFERENCES}

[1] Department of Energy, "Department of Energy; Buildings Energy Data Book" - http://buildingsdatabook.eren.doe.gov.

[2] Harle, Robert K., and Andy Hopper. "The potential for location-aware power management." Proceedings of the 10th international conference on Ubiquitous computing. ACM, 2008.

[3] Johnson Controls - http://www.johnsoncontrols.com/content/ us/en/products/building_efficiency/building_management.html

[4] Siemens Building Technologies http://www.buildingtechnologies.siemens.com

[5] Niagara AX - http://www.www.niagaraax.com

[6] Ur, Blase, et al. "Practical trigger-action programming in the smart home." Proceedings of the $32 \mathrm{nd}$ annual ACM conference on Human factors in computing systems. ACM, 2014.

[7] Dahl, Yngve, and Reidar-Martin Svendsen. "End-user composition interfaces for smart environments: A preliminary study of usability factors." Design, User Experience, and Usability. Theory, Methods, Tools and Practice. Springer Berlin Heidelberg, 2011. 118-127.

[8] Davidoff, Scott, et al. "Principles of smart home control." UbiComp 2006: Ubiquitous Computing. Springer Berlin Heidelberg, 2006. 19-34.

[9] Sohn, Timothy, and Anind Dey. "iCAP: an informal tool for interactive prototyping of context-aware applications." CHI'03 extended abstracts on Human factors in computing systems. ACM, 2003.

[10] Litvinova, Evgenia, and Petri Vuorimaa. "Engaging end users in real smart space programming." Proceedings of the 2012 ACM Conference on Ubiquitous Computing. ACM, 2012.

[11] Newman, Mark W., Ame Elliott, and Trevor F. Smith. "Providing an integrated user experience of networked media, devices, and services through end-user composition." Pervasive Computing. Springer Berlin Heidelberg, 2008. 213-227.

[12] Truong, Khai N., Elaine M. Huang, and Gregory D. Abowd. "CAMP: A magnetic poetry interface for end-user programming of capture applications for the home." UbiComp 2004: Ubiquitous Computing. Springer Berlin Heidelberg, 2004. 143-160.

[13] Nacci, A. A., et al. "A SystemC-based framework for the simulation of appliances networks in energy-aware smart spaces." Internet of Things (WF-IoT), 2014 IEEE World Forum on. IEEE, 2014.

[14] European Institute for Innovation and Technology ICT Labs. http://www.eitictlabs.eu/innovation- areas/smart-spaces/. Accessed: 26/06/2014

[15] W. Xie, Y. Shi, G. Xu, and Y. Mao. Smart platform - a software infrastructure for smart space (siss). In Fourth IEEE International Conference on Multimodal Interfaces, pages 429-434, 2002.

[16] E. Costanza, S. D. Ramchurn, and N. R. Jennings. Understanding domestic energy consumption through interactive visualisation: a field study. In Proceedings of the 2012 ACM Conference on Ubiquitous Computing, UbiComp '12, pages 216-225, New York, NY, USA, 2012. ACM.

[17] Y. Riche, J. Dodge, and R. A. Metoyer. Studying always-on electricity feedback in the home. In Proceedings of the SIGCHI Conference on Human Factors in Computing Systems, CHI '10, pages 1995-1998, New York, NY, USA, 2010. ACM. 